\title{
Nonlinear state estimation for rigid-body motion with low-pass sensors is
}

\author{
Henrik Rehbinder*, Xiaoming Hu \\ Division of Optimization and Systems Theory, Royal Institute of Technology, 10044 Stockholm, Sweden
}

Received 20 November 1998; received in revised form 25 June 1999; accepted 31 January 2000

\begin{abstract}
In this paper, we consider the state estimation problem for the nonlinear kinematic equations of a rigid body observed under low-pass sensors. The problem is motivated from a walking robot application where inclinometers and gyros are the sensors used. We show that a non-local high gain observer exists for the nonlinear rigid-body kinematic equations and that it under a small angle assumption is possible to use one inclinometer only to estimate two angles. (c) 2000 Elsevier Science B.V. All rights reserved.
\end{abstract}

Keywords: Nonlinear state estimation; Rigid body motion; Linear time-varying systems; Exponential observers; Inclinometers; Rate gyros

\section{Introduction}

In this paper, we consider the state estimation problem for the nonlinear kinematic equations of a rigid body observed under low-pass sensors. The rigid-body state estimation problem is highly motivated from various applications of which walking robots for difficult terrain is one. For such a robot, a reliable estimate of the trunks attitude is of paramount importance for the controller. With attitude we refer to pitch and roll and these quantities constitutes the robots sense of balance.

Rigid-body state estimation is the basis for inertial navigation systems for airplanes and has therefore received a lot of attention from both industry and the scientific community. Navigation systems typically rely on rate gyros of very high-quality which are integrated

\footnotetext{
th This work was sponsored in part by SSF through the Centre for Autonomous Systems and in part by TFR.

* Corresponding author.

E-mail addresses: henrikr@math.kth.se (H. Rehbinder), hu@ math.kth.se (X. Hu).
}

to get the airplanes orientation. Such high-quality gyros are very expensive and the robotics community has shown a lot of interest in using cheap gyros in conjunction with other complementary sensors to solve the problem. The idea is to fuse low-cost gyros with for example inclinometers to achieve a cheap and reliable solution.

The literature covering the use of high-quality gyros for rigid-body state estimation is vast and we will review papers relevant to our problem, that is papers that treat how to combine gyros and inclinometers and/or accelerometers. These papers study the problem from the perspective of various disciplines such as aircraft technology, mobile robotics, biomechanics and virtual reality. Baerveldt and Klang have studied the problem of fusing inclinometers and gyros using a frequency domain approach [1]. Their state estimator takes the dynamic characteristics of the inclinometer into account and is shown to be well performed as well as theoretically justifiable. However, only plane rotations are considered for which the problem is 
linear. Sakaguchi et al. describe a method for estimating human arm motions using gyros and accelerometers [11]. The working principle relies on being able to calculate the accelerations based on the joint angles in an arm model. A similar approach is used when Fuke et al. describe an attitude estimation system for a lunar rover [7]. Both Greene [8] and Foxlin [5,6] use an approach where the information from gyros, accelerometers and/or inclinometers are fused. The applications are aircraft systems and virtual reality helmets.

where $R \in \mathrm{SO}(3)$ is the coordinate transformation relating a body-fixed frame $(B)$ to an inertial system $(N)$ according to $x^{B}=R x^{N} . S(\omega)$ is the skew-symmetric wedge matrix

$S(\omega)=\left[\begin{array}{ccc}0 & \omega_{3} & -\omega_{2} \\ -\omega_{3} & 0 & \omega_{1} \\ \omega_{2} & -\omega_{1} & 0\end{array}\right]$

where $\omega_{i}$ are the components of the angular velocity vector expressed in the body-fixed frame. A parameterization of $\mathrm{SO}(3)$ suitable for this application is the yaw-pitch-roll parameterization

$R=\left[\begin{array}{ccc}\cos \theta_{3} \cos \theta_{1} & \sin \theta_{3} \cos \theta_{1} & -\sin \theta_{1} \\ -\sin \theta_{3} \cos \theta_{2}+\cos \theta_{3} \sin \theta_{1} \sin \theta_{2} & \cos \theta_{3} \cos \theta_{2}+\sin \theta_{3} \sin \theta_{1} \sin \theta_{2} & \cos \theta_{1} \sin \theta_{2} \\ \sin \theta_{3} \sin \theta_{2}+\cos \theta_{3} \sin \theta_{1} \cos \theta_{2} & \sin \theta_{3} \sin \theta_{1} \cos \theta_{2}-\cos \theta_{3} \sin \theta_{2} & \cos \theta_{1} \cos \theta_{2}\end{array}\right]$,

All the papers consider problems and sensors closely related to ours. There are however, interesting theoretical issues that are not addressed: for a walking robot, or for any terrain vehicle, rotations can typically be complex and truly 3 DOF in the sense that the motion is composed by simultaneous changes of yaw, pitch and roll. As the underlying kinematic equations for such motions are nonlinear they generate much more complicated mathematical problems than plane rotations. In the papers mentioned, either only plane rotations are considered, or there is no theoretical justification for the methods proposed. Convergence issues are for example not addressed.

The main contributions of this study is that it provides a proof for that it is theoretically possible to estimate a rigid body's attitude with inclinometers and gyros. The proposed observer is an exponential high-gain observer. We would like to stress that the convergence is non-local and that we are able to give an explicit expression for the domain of attraction. We also consider a simplified version of the same estimation problem when only one inclinometer measurement is available. Here we consider only small angles and show exponential convergence for a generic observer for time-varying stable linear systems. Finally, simulations illustrating the results are given.

\section{Problem formulation}

Consider the kinematics for a rotating rigid body which can be found in standard textbooks [9] and are

$\dot{R}=S(\omega) R$, defining local coordinates around $R=I$. Here we have used the notation $\theta_{1}$ for pitch, $\theta_{2}$ for roll and $\theta_{3}$ for yaw. This parameterization is suitable for many applications as the angles have an intuitive meaning and also, typical motions for mobile land robots are such that $\left|\theta_{1}\right|,\left|\theta_{2}\right|<\pi / 2$ for which the parameterization is unique. In these coordinates, the kinematics (1) are given by

$\dot{\theta}_{1}=\omega_{2} \cos \theta_{2}-\omega_{3} \sin \theta_{2}$,

$\dot{\theta}_{2}=\omega_{1}+\omega_{2} \sin \theta_{2} \tan \theta_{1}+\omega_{3} \cos \theta_{2} \tan \theta_{1}$,

where we only consider pitch $\left(\theta_{1}\right)$ and roll $\left(\theta_{2}\right)$.

The sensors at hand are the rate gyro measuring the angular velocity $\omega$ and the inclinometers measuring $\theta_{1}$ and $\theta_{2}$. Inclinometers can be modeled as first-order low-pass filters

$\dot{\eta}_{1}=\tau_{1}\left(\theta_{1}-\eta_{1}\right)$

$\dot{\eta}_{2}=\tau_{2}\left(\theta_{2}-\eta_{2}\right)$

where $\tau_{i}=1 / T_{i}$ are the inverse time constant and where $\eta_{i}$ are the inclinometer outputs. Introducing $\theta=$ $\left[\theta_{1}, \theta_{2}\right]^{\mathrm{T}}$ and $\eta=\left[\eta_{1}, \eta_{2}\right]^{\mathrm{T}}$ the system can be written as $\dot{\theta}=m(\theta) \omega$ $\dot{\eta}=\tau \theta-\tau \eta$

$y=C\left[\begin{array}{l}\theta \\ \eta\end{array}\right]$,

where

$m(\theta)=\left[\begin{array}{ccc}0 & \cos \theta_{2} & -\sin \theta_{2} \\ 1 & \sin \theta_{2} \tan \theta_{1} & \cos \theta_{2} \tan \theta_{1}\end{array}\right]$

and

$\tau=\operatorname{diag}\left(\tau_{1}, \tau_{2}\right)$ 
and

$C=\left[\begin{array}{ll}0 & I\end{array}\right]$.

Finally, by letting $x=\left[\theta^{\mathrm{T}}, \eta^{\mathrm{T}}\right]^{\mathrm{T}}$, we can write the system in the standard form

$\dot{x}=f(x, \omega)$,

$y=C x$.

In this paper, we consider the problem of reconstructing pitch and roll from sensor measurements. Throughout the paper, we assume that the measurements for $\omega$ are accurate enough, therefore they will be considered as known time-varying inputs to the pitch-and-roll equations.

\section{Observers with two inclinometers}

In this section, we construct an observer for the case where both pitch and roll are measured. Using a high-gain approach we show how to design an exponential observer that covers the range up to $\pi / 6$ for the absolute value of the pitch angle. This is, of course, not a local result. We make the following assumption.

\section{Assumption 3.1.}

$\left|\theta_{1}(t)\right| \leqslant \pi / 6-\delta / 3$,

$\left|\omega_{i}(t)\right| \leqslant \omega_{i}^{m} \quad$ for some $\omega_{i}^{m}>0, \quad i=2,3$,

where $\delta$ is any positive constant $<\pi / 2$.

For our walking robot that is under development now [12], we expect our control algorithms would keep the pitch in the range $-\pi / 6<\theta_{1}<\pi / 6$. Further it is not very conservative to assume that angular velocities are bounded. This assumption is therefore motivated from our application.

Theorem 3.1. Consider an observer for (5)

$\dot{\hat{x}}=f(\hat{x}, \omega)+L(y-C \hat{x})$,

$\hat{x}(0)=\left[\begin{array}{c}0 \\ \eta(0)\end{array}\right]$,

where $\hat{x}=\left(\hat{\theta}^{\mathrm{T}} \hat{\eta}^{\mathrm{T}}\right)^{\mathrm{T}}$ is the state estimate and let Assumption 3.1 be valid. Then, for each $\delta \in(0, \pi / 2)$ there is a $4 \times 2$ matrix $L$ such that the observer (7) is exponentially convergent.

The initial value $\hat{\eta}(0)=\eta(0)$ is not very restrictive as $\eta$ is the inclinometer output which we have access to.
Proof of Theorem 3.1. Let $L=\left[\begin{array}{ll}L_{1}^{\mathrm{T}} & L_{2}^{\mathrm{T}}\end{array}\right]^{\mathrm{T}}$. We will later (9) show how $L$ can be chosen properly. Introduce the estimation errors $\tilde{\theta}=\theta-\hat{\theta}, \tilde{\eta}=\eta-\hat{\eta}$ and consider the error dynamics which can be written as

$\dot{\tilde{\theta}}=\tilde{m}(\theta, \hat{\theta}, \omega)-L_{1} \tilde{\eta}$,

$\dot{\tilde{\eta}}=\tau \tilde{\theta}-\left(\tau+L_{2}\right) \tilde{\eta}$,

where the nonlinear terms are given by

$$
\begin{aligned}
\tilde{m}(\theta, \hat{\theta}, \omega)= & {\left[\begin{array}{c}
\cos \hat{\theta}_{2}-\cos \theta_{2} \\
\sin \hat{\theta}_{2} \tan \hat{\theta}_{1}-\sin \theta_{2} \tan \theta_{1}
\end{array}\right] \omega_{2} } \\
& +\left[\begin{array}{c}
-\sin \hat{\theta}_{2}+\sin \theta_{2} \\
\cos \hat{\theta}_{2} \tan \hat{\theta}_{1}-\cos \theta_{2} \tan \theta_{1}
\end{array}\right] \omega_{3} .
\end{aligned}
$$

Defining

$A=\left[\begin{array}{cc}0 & -L_{1} \\ \tau & -\left(\tau+L_{2}\right)\end{array}\right]$

and

$L=\left[\begin{array}{l}L_{1} \\ L_{2}\end{array}\right]=\left[\begin{array}{cc}-c_{1} & 0 \\ 0 & -c_{2} \\ -\tau_{1}-l_{1} & 0 \\ 0 & -\tau_{2}-l_{2}\end{array}\right]$,

the error dynamics (8) can be written as

$\dot{\tilde{x}}(t)=A \tilde{x}(t)+\left[\begin{array}{c}\tilde{m}(\theta(t), \hat{\theta}(t), \omega(t)) \\ 0\end{array}\right]$,

where $\tilde{x}=\left[\begin{array}{ll}\tilde{\theta}^{\mathrm{T}} & \tilde{\eta}^{\mathrm{T}}\end{array}\right]^{\mathrm{T}}$. If we partition the transition matrix $\Phi(t)=\mathrm{e}^{A t}$ associated with $A$ as

$\Phi(t)=\left[\begin{array}{ll}\Phi_{11}(t) & \Phi_{12}(t) \\ \Phi_{21}(t) & \Phi_{22}(t)\end{array}\right]$

and recall that

$\tilde{\eta}(0)=0$.

Then by integrating (8) and using (10) and (11) we can write

$$
\left[\begin{array}{l}
\tilde{\theta} \\
\tilde{\eta}
\end{array}\right](t)=\left[\begin{array}{l}
\Phi_{11}(t) \tilde{\theta}(0) \\
\Phi_{21}(t) \tilde{\theta}(0)
\end{array}\right]+\int_{0}^{t}\left[\begin{array}{l}
\Phi_{11}(t-s) \tilde{m} \\
\Phi_{21}(t-s) \tilde{m}
\end{array}\right] \mathrm{d} s .
$$

It is now straightforward to show that if we choose $l_{i}=3 l$ and $c_{i}=-2 l^{2} / \tau_{i}$, for some gain $l>0$ then

$$
\begin{aligned}
& \Phi_{11}(t)=\left(2 \mathrm{e}^{-l t}-\mathrm{e}^{-2 l t}\right) I, \\
& \Phi_{21}(t)=\frac{1}{l}\left(\mathrm{e}^{-l t}-\mathrm{e}^{-2 l t}\right) \operatorname{diag}\left(\tau_{1}, \tau_{2}\right),
\end{aligned}
$$

which are bounded by

$$
\begin{aligned}
& \left\|\Phi_{11}(t)\right\|_{\infty} \leqslant 2 \mathrm{e}^{-l t}, \\
& \left\|\Phi_{21}(t)\right\|_{\infty} \leqslant \max \left(\tau_{1}, \tau_{2}\right) \mathrm{e}^{-l t}, \quad l>1,
\end{aligned}
$$

where the norm used is the maximum norm. 
To establish bounds on $\tilde{m}$, we first have to show that, with our choice of the initial condition $\left(\hat{\theta}_{1}(0)=0\right)$, it will hold that

$\left|\tilde{\theta}_{1}(t)\right| \leqslant \frac{\pi}{3}-\frac{2 \delta}{3}$

for all $t \geqslant 0$ if $l$ is sufficiently large. From (12) we have that

$$
\begin{aligned}
\tilde{\theta}_{1}(t)= & \left(2 \mathrm{e}^{-l t}-\mathrm{e}^{-2 l t}\right) \tilde{\theta}_{1}(0) \\
& +\int_{0}^{t}\left(2 \mathrm{e}^{-l(t-s)}-\mathrm{e}^{-2 l(t-s)}\right) \tilde{m}_{1} \mathrm{~d} s,
\end{aligned}
$$

where $\tilde{m}_{1}=\left(\cos \hat{\theta}_{2}-\cos \theta_{2}\right) \omega_{2}+\left(-\sin \hat{\theta}_{2}+\sin \theta_{2}\right) \omega_{3}$ which is bounded by $\left|\tilde{m}_{1}\right| \leqslant 2\left(\omega_{2}^{m}+\omega_{3}^{m}\right)$. Using this we have

$\left|\tilde{\theta}_{1}(t)\right| \leqslant 2 \mathrm{e}^{-l t}\left|\tilde{\theta}_{1}(0)\right|+\frac{4}{l}\left(\omega_{2}^{m}+\omega_{3}^{m}\right)\left(1-\mathrm{e}^{-l t}\right)$.

It is now a matter of straightforward calculations to see that if we take

$l>\frac{4\left(\omega_{2}^{m}+\omega_{3}^{m}\right)}{\pi / 6-\delta / 3}$

then the fact that $\left|\tilde{\theta}_{1}(0)\right|<\pi / 6-\delta / 3$ by Assumption 3.1 guarantees that

$\left|\tilde{\theta}_{1}(t)\right| \leqslant \frac{\pi}{3}-\frac{2 \delta}{3}$.

To proceed with bounding $\tilde{m}$ we use the mean value theorem stating that, for $\mathrm{C}^{1}$-functions, $g(z+a)-g(z)=$ $g^{\prime}(z+\alpha a) a$ where $\alpha \in[0,1]$. For $\tilde{m}$ this gives

$$
\begin{aligned}
& \|\tilde{m}\|_{\infty} \leqslant\left(\omega_{2}^{m}+\omega_{3}^{m}\right) \\
& \quad\left(\left|\tan \left(\theta_{1}+\alpha \tilde{\theta}\right)\right|+\frac{1}{\cos ^{2}\left(\theta_{1}+\alpha \tilde{\theta}\right)}\right)\|\tilde{\theta}(t)\|_{\infty}
\end{aligned}
$$

for some $\alpha \in[0,1]$. Now, using (19) and Assumption 3.1 it is clear that $\left|\theta_{1}+\alpha \tilde{\theta}\right|<\pi / 2-\delta$ and thus $\left(\omega_{2}^{m}+\omega_{3}^{m}\right)\left(\left|\tan \left(\theta_{1}+\alpha \tilde{\theta}\right)\right|+1 / \cos ^{2}\left(\theta_{1}+\alpha \tilde{\theta}\right)\right)<\left(\omega_{2}^{m}+\right.$ $\left.\omega_{3}^{m}\right)\left(|\tan (\pi / 2-\delta)|+1 / \cos ^{2}(\pi / 2-\delta)\right)=K<\infty$. We thus have

$\|\tilde{m}\|_{\infty} \leqslant K\|\tilde{\theta}(t)\|_{\infty}$.

To show exponential convergence, we use (12), (14) and (20) to show that

$$
\begin{aligned}
\|\tilde{\theta}(t)\|_{\infty} \leqslant & 2 \mathrm{e}^{-l t}\|\tilde{\theta}(0)\|_{\infty} \\
& +\int_{0}^{t} 2 \mathrm{e}^{-l(t-s)} K\|\tilde{\theta}(s)\|_{\infty} \mathrm{d} s, \\
\|\tilde{\eta}(t)\|_{\infty} \leqslant & \tau_{m} \mathrm{e}^{-l t}\|\tilde{\theta}(0)\|_{\infty} \\
& +\int_{0}^{t} \tau_{m} \mathrm{e}^{-l(t-s)} K\|\tilde{\theta}(s)\|_{\infty} \mathrm{d} s,
\end{aligned}
$$

where $\tau_{m}=\max \left(\tau_{1}, \tau_{2}\right)$. Now, define $p(t)=$ $\mathrm{e}^{l t}|| \tilde{\theta}(t) \|_{\infty}$. The first of equations (21) gives

$p(t) \leqslant 2\|\tilde{\theta}(0)\|_{\infty}+\int_{0}^{t} 2 K p(s) \mathrm{d} s$

and using Gronwall-Bellmans lemma we obtain $p(t) \leqslant 2\|\tilde{\theta}(0)\|_{\infty} \mathrm{e}^{2 K t}$.

We now have an exponential bound on the $\theta$-errors

$\|\tilde{\theta}(t)\|_{\infty} \leqslant 2|| \tilde{\theta}(0) \|_{\infty} \mathrm{e}^{-(l-2 K) t}$,

and for $l>2 K$ these errors exponentially tend to zero. Using (22), it can be shown that also $\tilde{\eta}$ is exponentially bounded by

$$
\|\tilde{\eta}(t)\| \leqslant \frac{\tau_{m}\|\tilde{\theta}(0)\|_{\infty}}{K}\left((K-1) \mathrm{e}^{-l t}+\mathrm{e}^{-(l-2 K) t}\right) .
$$

In summary, we have now shown that if $l>$ $\max \left(1,2 K, 4\left(\omega_{2}^{m}+\omega_{3}^{m}\right) /(\pi / 6-\delta / 3)\right)$, the observer (7) converges provided Assumption 3.1 holds, which obviously is a non-local result.

\section{Observers with one inclinometer}

In this section, we consider the problem of reconstructing both pitch and roll in the case where only one inclinometer is available. Without loss of generality, we assume the inclinometer for pitch is available. Solving this problem would be interesting if one of the inclinometers becomes unreliable due to malfunction, or as a result of impact in one direction.

For this problem, we only consider the case of small angles. Then the nonlinear dynamics can be simplified as

$\dot{\theta}_{1}=-\omega_{3}(t) \theta_{2}+\omega_{2}$,

$\dot{\theta}_{2}=\omega_{3}(t) \theta_{1}+\omega_{1}$,

$\dot{\eta}_{1}=\tau \theta_{1}-\tau \eta_{1}$

which is linear but time varying.

We show now that under some rather mild conditions there exist exponential observers for the system. Let us first rewrite (24) into the matrix form:

$\dot{x}=A(t) x+B \omega$,

$y=C x$,

where

$x=\left[\theta_{1}, \theta_{2}, \eta_{1}\right]^{\mathrm{T}}, \quad C=\left[\begin{array}{lll}0 & 0 & 1\end{array}\right], \quad B=\left[\begin{array}{lll}0 & 1 & 0 \\ 1 & 0 & 0\end{array}\right]$ 
and where

$A(t)=\left[\begin{array}{ccc}0 & -\omega_{3}(t) & 0 \\ \omega_{3}(t) & 0 & 0 \\ \tau & 0 & -\tau\end{array}\right]$.

The following result of ours states that it is possible to estimate the attitude using only one inclinometer:

Theorem 4.1. Suppose $\omega_{3}(t)$ is such that the following observability condition is satisfied:

$\int_{0}^{T} \Phi^{*}(t+s, t) C^{*} C \Phi(t+s, t) \mathrm{d} s \geqslant \varepsilon I$

for some $\varepsilon>0, T>0$ and any $t \geqslant 0$ where $\Phi(t, s)$ is the transition matrix of $A(t)$. Then there exists an exponential observer in the following form:

$\dot{\hat{x}}=A(t) \hat{x}-L(t)(C \hat{x}-y(t))+B \omega$.

Remark. Eq. (27) is fulfilled for example by constant $\omega_{3} \neq 0$ or for $\omega_{3}=\sin (t)$.

Before we give a constructive proof for the theorem, we will show a general result for linear time-varying systems which, we believe, is new and interesting in its own right and will serve as the basis for our proof of Theorem 4.1. There are quite classical results on the convergence of time-varying Kalman filters [3] which can of course be adopted to the deterministic case. However, those results require the solution of a time-varying Riccati equation and the proof is neither easy to find, nor to read. As is well known, the time-varying Riccati equation is hard to integrate (see, for example, [10, pp. 257-261]) and we therefore choose to use our proposed observer for time-varying systems in which one only needs to solve a Lyapunov equation. A somewhat similar observer is proposed in [4] which uses a gradient algorithm-based method. In that paper however, the system is assumed to be Poisson stable, which is not the case for $A(t)$ defined in (26) (see, for example, [9, p. 288] for definition). As we only assume critical stability, the observer presented below is more general.

\section{Proposition 4.1. Consider}

$\dot{x}=A(t) x$,

$y=C x$.

If there exists a $P(\cdot):[0, \infty) \rightarrow \mathbb{R}^{n \times n}$, with the properties that $0<m I \leqslant P(t) \leqslant M I$ and

$A^{*}(t) P(t)+P(t) A(t)+\dot{P}(t) \leqslant 0$ and there exist $\varepsilon>0$ and $T>0$ such that for any $t \geqslant 0$

$\int_{0}^{T} \Phi^{*}(t+s, t) C^{*} C \Phi(t+s, t) \mathrm{d} s \geqslant \varepsilon I$,

where $\Phi(t, s)$ is the transition matrix of $A(t)$, then

$\dot{\hat{x}}=A(t) \hat{x}-P(t)^{-1} C^{*}(C \hat{x}-y(t))$

is an observer with exponentially decaying error.

We will prove the proposition at the end of the section. Let us first use it to show Theorem 4.1.

Proof of Theorem 4.1. Since the term $B \omega$ in (25) is known and independent of the states, we can without loss of generality take it as 0 . Now, we can use Proposition 4.1. Let

$P(t)=I+\left[\begin{array}{cc}\alpha(t) \alpha(t)^{*} & -\alpha(t) \\ -\alpha(t)^{*} & 0\end{array}\right]$,

where $\alpha(t)$ satisfies

$\dot{\alpha}=\left[\begin{array}{cc}-\tau & -\omega_{3}(t) \\ \omega_{3}(t) & -\tau\end{array}\right] \alpha+\left[\begin{array}{l}\tau \\ 0\end{array}\right]$,

$\alpha(0)=0$.

A straightforward calculation gives that

$V(x, t)=x^{\mathrm{T}} P(t) x$

is a Lyapunov function for (24), or in other words,

$\dot{P}(t)+A(t) P(t)+P(t) A^{*}(t) \leqslant 0$.

Now, we only need to show $P(t)$ is bounded below and above:

$m I<P(t)<M I$.

To show this, note that (30) has a Lyapunov function $V_{1}(x)=\|x\|^{2}$ with $\dot{V}_{1}=-2 \tau\|x\|^{2}$ and thus is exponentially stable. Here we use the Euclidean norm. Therefore, $\alpha(t)$ is bounded and as a consequence, $P(t)$ is bounded above. To show that $P(t)$ is bounded below we consider the eigenvalues. It is tedious but straightforward to find the minimal eigenvalue

$\lambda_{\min }=1+\frac{\|\alpha\|^{2}-\sqrt{\|\alpha\|^{4}+4\|\alpha\|^{2}}}{2}$

for which it holds that $\lambda_{\min }>0$ and for bounded $\alpha$, $\lambda_{\text {min }} \geqslant \varepsilon>0$.

Therefore, the hypotheses of Proposition 4.1 are satisfied and which implies, with the setting $L(t)=$ $P(t)^{-1} C^{*}$, the observer of Theorem 4.1 is an exponential observer, when the observability condition is fulfilled. 
Now we prove Proposition 4.1. Before we proceed with the proof, we need the following two lemmas [2].

Lemma 4.1. Let $K(\cdot) \in L_{\infty}\left((0,+\infty) ; \mathbb{R}^{n \times n}\right)$. Consider the system

$\dot{z}=K(t) z$.

Assume that

$\int_{t_{0}}^{\infty}|z(t)|^{2} \mathrm{~d} t \leqslant c^{2}\left|z\left(t_{0}\right)\right|^{2}$

for any solution $z(\cdot)$ of (32) and any $t_{0} \geqslant 0$ with the constant $c>0$ being independent of $z(\cdot)$ and $t_{0}$.

Then and only then

$|z(t)| \leqslant b\left|z\left(t_{0}\right)\right| \mathrm{e}^{-r\left(t-t_{0}\right)}$

for some $b>0, r>0$.

Lemma 4.2. Let $\phi(\cdot) \in L_{2}\left(\left[t_{0},+\infty\right) ; \mathbb{R}^{n}\right)$ and $\theta>0$. Denote

$\bar{\phi}(t):=\int_{0}^{\theta} \phi(t+s) \mathrm{d} s \quad \forall t \geqslant t_{0}$.

Then, $\bar{\phi}(\cdot) \in L_{2}\left(\left[t_{0},+\infty\right) ; \mathbb{R}^{n}\right)$ and

$|\bar{\phi}(\cdot)|_{2} \leqslant \theta|\phi(\cdot)|_{2}$.

Proof. By using the Cauchy-Schwartz inequality, we get

$|\bar{\phi}(t)| \leqslant \int_{t}^{t+\theta} 1 \times|\phi(s)| \mathrm{d} s \leqslant \sqrt{\theta}\left(\int_{t}^{t+\theta}|\phi(s)|^{2} \mathrm{~d} s\right)^{1 / 2}$.

Hence, by changing the order of integration,

$$
\begin{gathered}
\int_{t_{0}}^{\infty}|\bar{\phi}(t)|^{2} \mathrm{~d} t \leqslant \theta \int_{t_{0}}^{\infty}\left(\int_{t}^{t+\theta}|\phi(s)|^{2} \mathrm{~d} s\right) \mathrm{d} t \\
=\theta \int_{t_{0}}^{\infty}|\phi(s)|^{2} \mathrm{~d} s \int_{s-\theta}^{s} \mathrm{~d} t=\theta^{2}|\phi(\cdot)|_{2}^{2} .
\end{gathered}
$$

Thus, $\bar{\phi}(\cdot) \in L_{2}$ and (35) is true.

Now we are ready to prove the proposition.

Proof. Denote

$z=x(t)-\hat{x}$.

Then,

$\dot{z}=A z-P^{-1} C^{*} C z$

and

$$
\begin{aligned}
\frac{\mathrm{d}}{\mathrm{d} t}\left(z^{*} P z\right) & =z^{*}\left(P A+A^{*} P+\dot{P}\right) z-2 z^{*} C^{*} C z \\
& \leqslant-2|C z|^{2} .
\end{aligned}
$$

So, for any two instants $t \geqslant t_{0} \geqslant 0$,

$z(t)^{*} P(t) z(t) \leqslant z\left(t_{0}\right)^{*} P\left(t_{0}\right) z\left(t_{0}\right)-2 \int_{t_{0}}^{t}|C z(s)|^{2} \mathrm{~d} s$.

Therefore,

$z(t)^{*} P(t) z(t) \leqslant z\left(t_{0}\right)^{*} P\left(t_{0}\right) z\left(t_{0}\right) \leqslant M\left|z\left(t_{0}\right)\right|^{2}$

and

$2 \int_{t_{0}}^{t}|C z(s)|^{2} \mathrm{~d} s \leqslant M\left|z\left(t_{0}\right)\right|^{2}$.

By assumption $a^{*} P a \geqslant m|a|^{2}$, so we have

$m|z(t)|^{2} \leqslant z(t)^{*} P(t) z(t) \leqslant M\left|z\left(t_{0}\right)\right|^{2}$,

$|z(t)| \leqslant \sqrt{\frac{M}{m}}\left|z\left(t_{0}\right)\right|$

and

$\int_{t_{0}}^{\infty}|C z(s)|^{2} \mathrm{~d} s \leqslant \frac{M}{2}\left|z\left(t_{0}\right)\right|^{2}$.

Solving the error equation we have

$z(t+s)=\Phi(t+s, t) z(t)-\int_{t}^{t+s} \phi(t+s, \theta) \zeta(\theta) \mathrm{d} \theta$,

where $\zeta(t)=P^{-1} C^{*} C z(t)$. We denote $\varphi_{s}(t)=$ $\int_{t}^{t+s} \phi(t+s, \theta) \zeta(\theta) \mathrm{d} \theta$. Let $t, s \geqslant 0, s \leqslant T$, where $T$ is the constant from the observability condition. Since the matrix $A(t)$ is Lyapunov stable, $\|\Phi(t+\tau, t)\| \leqslant \beta<\infty$ for all $\tau \geqslant 0$. So, in (39),

$\left|\varphi_{s}(t)\right| \leqslant \beta \int_{t}^{t+T}|\zeta(\theta)| \mathrm{d} \theta:=\chi(t)$,

where by Lemma 4.2,

$|\chi(\cdot)|_{2} \leqslant \beta T|\zeta(\cdot)|_{2} \leqslant \beta \frac{1}{m}\left\|C^{*}\right\| \sqrt{\frac{M}{2}} T\left|z\left(t_{0}\right)\right|$.

Now we get

$$
\begin{aligned}
& \int_{0}^{T}|C \Phi(t+s, t) z(t)|^{2} \mathrm{~d} s \\
& \quad=\int_{0}^{T}\left|C\left(z(t+s)+\varphi_{s}(t)\right)\right|^{2} \mathrm{~d} s \\
& \quad \leqslant 2 \int_{0}^{T}|C z(t+s)|^{2} \mathrm{~d} s+2 \int_{0}^{T}\left|C \varphi_{s}(t)\right|^{2} \mathrm{~d} s .
\end{aligned}
$$



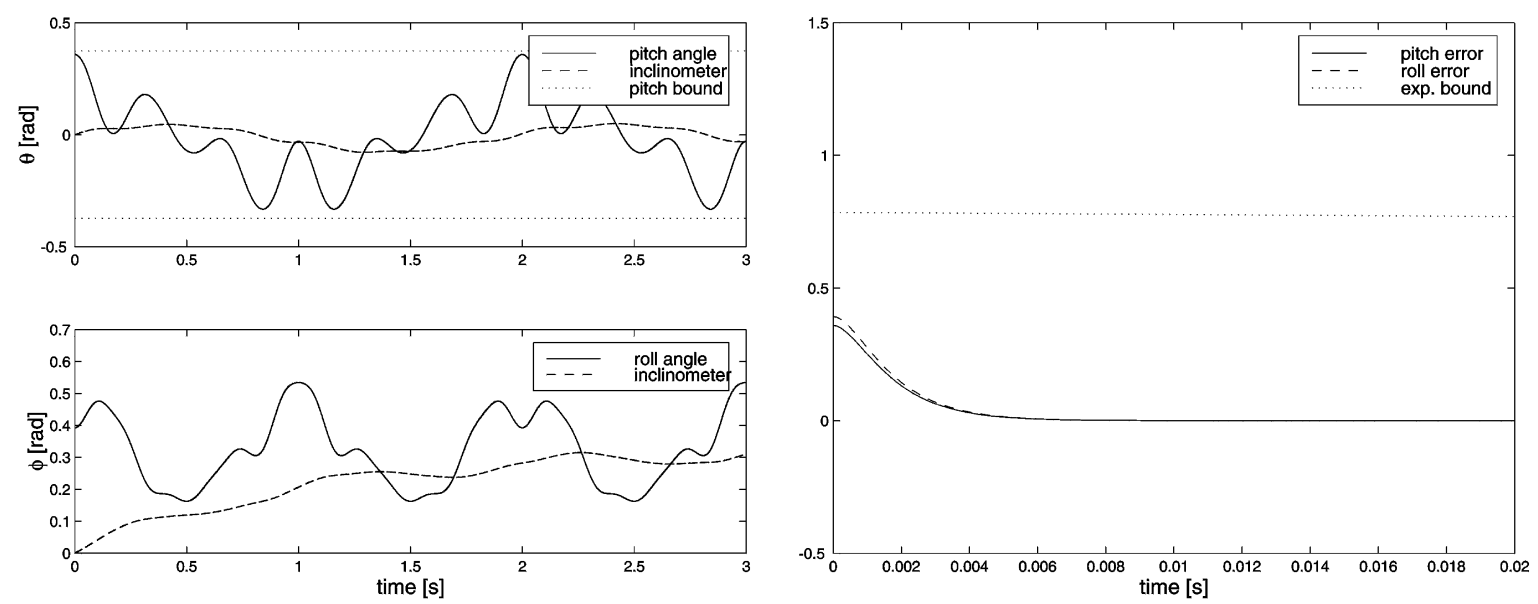

Fig. 1. Estimation with two inclinometers.

Then,

$$
\begin{aligned}
\int_{t_{0}}^{\infty} \mathrm{d} t \int_{0}^{T}|C \Phi(t+s, t) z(t)|^{2} \mathrm{~d} s \\
\leqslant 2 \int_{t_{0}}^{\infty} \mathrm{d} t\left(\int_{0}^{T}|C z(t+s)|^{2} \mathrm{~d} s+\int_{0}^{T}\left|C \varphi_{s}(t)\right|^{2} \mathrm{~d} s\right) \\
\leqslant 2 \int_{t_{0}}^{\infty}|C z(r)|^{2} \mathrm{~d} r \int_{\max \left\{t_{0}, r-T\right\}}^{r} \mathrm{~d} t \\
\quad+2 \int_{t_{0}}^{\infty} T|| C \mid \|^{2} \chi(t) \mathrm{d} t \\
\leqslant T M\left(1+\frac{T^{2}}{m^{2}} \beta^{2}|| C||^{4}\right)\left|z\left(t_{0}\right)\right|^{2} .
\end{aligned}
$$

On the other hand,

$$
\begin{aligned}
\int_{t_{0}}^{\infty} \mathrm{d} t \int_{0}^{T}|C \Phi(t+s, t) z(t)|^{2} \mathrm{~d} s \\
=\int_{t_{0}}^{\infty}\left(z ( t ) ^ { * } \left[\int_{0}^{T} \Phi^{*}(t+s, t)\right.\right. \\
\left.\left.C^{*} \Phi(t+s, t) C \mathrm{~d} s\right] z(t)\right) \mathrm{d} t .
\end{aligned}
$$

Then the observability hypothesis yields

$$
\int_{t_{0}}^{\infty} \mathrm{d} t \int_{0}^{T}|C \Phi(t+s, t) z(t)|^{2} \mathrm{~d} s \geqslant \varepsilon \int_{t_{0}}^{\infty}|z(t)|^{2} \mathrm{~d} t
$$

Thus,

$$
\int_{t_{0}}^{\infty}|z(t)|^{2} \mathrm{~d} t \leqslant \frac{T M}{\varepsilon}\left(1+\frac{T^{2}}{m^{2}} \beta^{2}\|C\|^{4}\right)\left|z\left(t_{0}\right)\right|^{2} .
$$

This means that the system (36) satisfies the hypotheses of Lemma 4.1. In other words, the assumptions of Lemma 4.1 are fulfilled with respect to the matrix function

$K(t):=A(t)-P(t)^{-1} C^{*} C$.

Therefore, the error tends to zero exponentially.

\section{Simulations}

\subsection{High-gain state estimation for rigid body motion}

To illustrate the results in Section 3, consider a rigid body (5) equipped with the sensors described in Section 2. Let it be subject to the angular velocities $\omega=$

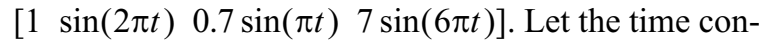
stants be given by $\tau_{i}=1$ and take for instance $\delta=0.15$ in Assumption 3.1. Then, $K=395.75$ and $l>800$ guarantees convergent estimates. Let the initial state be given by $\left[\theta_{1}, \theta_{2}, \eta_{1}, \eta_{2}\right](0)=[\pi / 6-1.1 \delta, \pi / 8,0,0]$. As can be seen in Fig. 1, our choice of initial values and angular velocities give pitch angles within the prescribed bounds. From the figure it is also obvious that the inclinometer output is not suitable for control purposes.

Applying the proposed high gain observer, the errors converge to zero as expected. For this case the convergence is considerably faster than the exponential bounds which also is shown in the figure. 

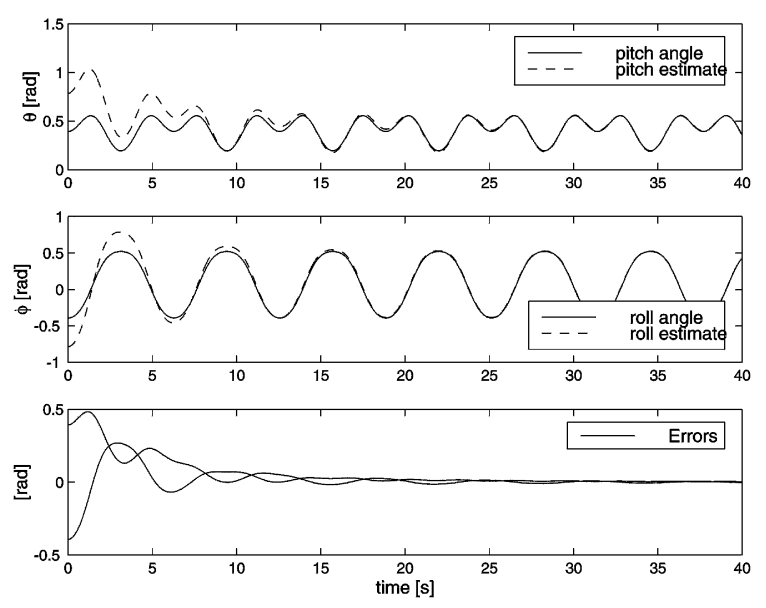

Fig. 2. Estimation with one inclinometer.

\subsection{State estimation with one inclinometer}

In Section 4 it was shown that, for small angles it is possible to estimate pitch and roll using only an inclinometer for the pitch angle. To illustrate this, we consider a motion generated by $\left[\begin{array}{lll}\theta_{1} & \theta_{2} & \eta_{1}\end{array}\right](0)=$ $[\pi / 8-\pi / 80]$ and $\omega=\left[\begin{array}{lll}0 & 0 & \sin t\end{array}\right]$. In Fig. 2, pitch, roll, estimates and errors are given. The observer is seen to converge.

\section{Summary}

We have studied the state-estimation problem for rigid bodies where the sensors used are inclinometers and gyros. The existence of an exponential observer of high-gain type is shown and we give an explicit expression for the domain of attraction. Further, we show that if one inclinometer is out of order or unreliable due to for example a sudden impact it is still possible to estimate pitch-and-roll given small angles and strong observability. Future work amounts to considering observers for noisy sensors such as extended Kalman filters and other sensor configurations. A rotating experimental platform equipped with sensors has been built which we will use to evaluate the algorithms performance in the real world. Finally, the sensor system will be implemented on a walking robot.

\section{Acknowledgements}

The authors would like to thank a referee for his careful reading of the manuscript and many suggestions for improving the presentation.

\section{References}

[1] A.J. Baerveldt, R. Klang, A low-cost and low-weight attitude estimation system for an autonomous helicopter, IEEE International Conference on Intelligent Engineering Systems, Proceedings, 1997, pp. 391-395.

[2] R. Brocket, Finite Dimensional Linear Systems, Wiley, New York, 1970.

[3] R.S. Bucy, The Ricatti equation and its bounds, J. Comput. System Sci. 6 (1972) 343-353.

[4] M.-S. Chen, Control of linear time-varying systems by the gradient algorithm, Proceedings of the 36th Conference on Decision and Control, San Diego, CA, IEEE, New York, December 1997.

[5] E. Foxlin, Inertial head-tracker sensor fusion by a complementary separate-bias Kalman filter, Proceedings of the IEEE 1996 Virtual Reality Annual International Symposium, 1996, pp. 185-94.

[6] E. Foxlin, M. Harrington, Y. Altshuler, Miniature 6-DOF inertial system for tracking HMD, Proceedings of the SPIE, Vol. 3362, 1998, pp. 214-228.

[7] Y. Fuke, E. Krotkov, Dead reckoning for a lunar rover on uneven terrain, Proceedings of the 1996 IEEE International Conference on Robotics and Automation, Minneapolis, MN, April 1996, pp. 411-416.

[8] M. Greene, A solid state attitude heading reference system for general aviation, Proceedings of the 1996 IEEE Conference on Emerging Technologies and Factory Automation, Vol. 2, 1996, pp. 413-17.

[9] A. Isidori, Nonlinear Control Systems, Springer, London, 1995.

[10] P.S. Maybeck, Stochastic Models, Estimation and Control, Vol. 1, Academic Press, New york, 1979.

[11] T. Sakaguchi, K. Tsutomu, H. Katayose, K. Sato, S. Inokuchi, Human motion capture by integrating gyroscopes and accelerometers, Proceedings of the 1996 IEEE/SICE/RSJ International Conference on Multisensor Fusion and Integration for Intelligent Systems, 1996, pp. 470-475.

[12] L. Wallentin, K. Jansson, S. Andersson, SLEIPNER3 - A four legged robot platform, in: K. Andersson, J.G. Persson (Eds.), NordDesign'98, Stockholm, Sweden, August 1998, pp. 289-297. 